\title{
Metagenomes and metatranscriptomes from the L4 long-term coastal monitoring station in the Western English Channel
}

\author{
Jack A. Gilbert, ${ }^{1,2,3^{*}}$ Folker Meyer, ${ }^{2,3}$ Lynn Schriml ${ }^{4}$, lan R Joint, ${ }^{1}$ Martin Mühling, ${ }^{5}$ Dawn \\ Field $^{6}$ \\ ${ }^{1}$ Plymouth Marine Laboratory, Prospect Place, Plymouth, PL1 3DH, U. \\ ${ }^{2}$ Argonne National laboratory, 9700 S. Cass Ave., Argonne, IL 60439, USA \\ ${ }^{3}$ University of Chicago, Chicago, IL 60637, USA \\ ${ }^{4}$ University of Maryland School of Medicine, 655 W. Baltimore Street, Baltimore MD \\ 21201 \\ ${ }^{5}$ TU Bergakademie Freiberg, IÖZ - Interdisciplinary Centre for Ecology, Leipziger Str. 29, \\ 09599 Freiberg, Germany. \\ ${ }^{6}$ NERC Centre for Ecology and Hydrology, Mansfield Road, Oxford, OX1 3SR, UK \\ *Corresponding author: gilbertjack@anl.gov
}

Keywords: Marine, aerobic, surface water, coastal, temperate, metagenome, metatranscriptome, pyrosequencing, time-series, diel, seasonal

Both metagenomic data and metatranscriptomic data were collected from surface water (0$2 \mathrm{~m}$ ) of the L4 sampling station $(50.2518 \mathrm{~N}, 4.2089 \mathrm{~W})$, which is part of the Western Channel Observatory long-term coastal-marine monitoring station. We previously generated from this area a six-year time series of 16S rRNA V6 data, which demonstrated robust seasonal structure for the bacterial community, with diversity correlated with day length. Here we describe the features of these metagenomes and metatranscriptomes. We generated 8 metagenomes (4.5 million sequences, $1.9 \mathrm{Gbp}$, average read-length $350 \mathrm{bp}$ ) and 7 metatranscriptomes (392,632 putative mRNA-derived sequences, $159 \mathrm{Mbp}$, average read-length $272 \mathrm{bp})$ for eight time-points sampled in 2008. These time points represent three seasons (winter, spring, and summer) and include both day and night samples. These data demonstrate the major differences between genetic potential and actuality, whereby genomes follow general seasonal trends yet with surprisingly little change in the functional potential over time; transcripts tended to be far more structured by changes occurring between day and night.

\section{Introduction}

The Western Channel Observatory station L4, located off the Plymouth coast in the UK, has been collecting environmental data for almost a century [1]. This includes published 16S rRNA V6 amplicon pyrosequencing data cataloging monthly patterns in microbial diversity [2,3]. The importance of the area rests with its being a transition zone between many northern and southern planktonic species [1] and with the fact that, as a major confluence between the North Atlantic Ocean and the North Sea, water masses exhibit extremely short residence times ( $>2$ months [4]; ). In the study reported here, we use shotgun metagenomics and metatranscriptomics to identify the relationship between genetic and functional diversity at station L4.

\section{Classification and features Relationship of reported datasets}

We generated 8 metagenomes and 7 metatranscriptomes for eight time points. Figure 1 shows the relationships of these metagenomes and metatranscriptomes; the figure was produced by using a group-average clustering dendrogram representing the relationships based on comparison of 66,529 amino acid sequences of greater than 40 amino acids predicted from each dataset (for details of the process, see Metagenome Annotation). One can 
clearly see that the metagenomic and metatranscriptomic data cluster separately. The metagenomic data shows an average similarity of less than $2 \%$, clustered by season, from which one can infer that the seasonal differences are stronger than the diel differences. On the other hand, the metatranscriptomes show more similarity and a tendency to cluster by diel time point; specifically, the April night data and January night data are more similar to each other than either is to the April day data and January day data. The August metatranscriptomes cluster by themselves, but this clustering is also structured by day and night. Table 1 details the classification and general features of the metagenomic datasets information for this study in MIMS format.

\section{Environmental characteristics and descriptions}

Environmental data was collected for temperature, density, salinity, chlorophyll a, total concentration of organic nitrogen and carbon, nitrate, ammonia, silicate, and phosphate [Table 2]. The methods used are described on the Western Channel Observatory website.

Figure 2 plots the environmental trends at L4 averaged for the years 2003-2008; the graph clearly shows the differences among the samples taken in the three months. Figure 3 shows a principal component analysis of the environmental parameters recorded during this study. Evident from the figure is the fact that the January samples have higher nutrient concentrations, the April samples show changes in the water salinity as a consequence of density, and the August samples show changes in temperature and chlorophyll a concentration.

\section{Metagenome sequencing and annotation Metagenome project history}

Two factors motivated the choice of station L4: its century-long history of environmental data [7] and the six years of $16 \mathrm{~S}$ rRNA V6 amplicon pyrosequencing information detailing microbial diversity patterns [2,3], from which we inferred interannual variability from our single-year study. All 16S rRNA V6 amplicon pyrosequencing data have been submitted to the NCBI short reads archive under SRA009436 and registered with the GOLD database (Gm00104). The data also can be accessed from the VAMPS server. The metagenomic data and metatranscriptomic data are available on the CAMERA website under Western Channel Observatory Microbial Metagenomic Study and on the Metagenome Rapid Annotation using Subsystem Technology (MG-RAST) system under 4443360-63, 4443365-68 and 4444077, 4445065-68, 4445070, 4445081, and 4444083, as well as through the INSDC short-reads archive under ERP000118. Table 1, Table 2, Table 3, and Table 4 detail the metagenomic sequencing project information for this study in MIMS format.

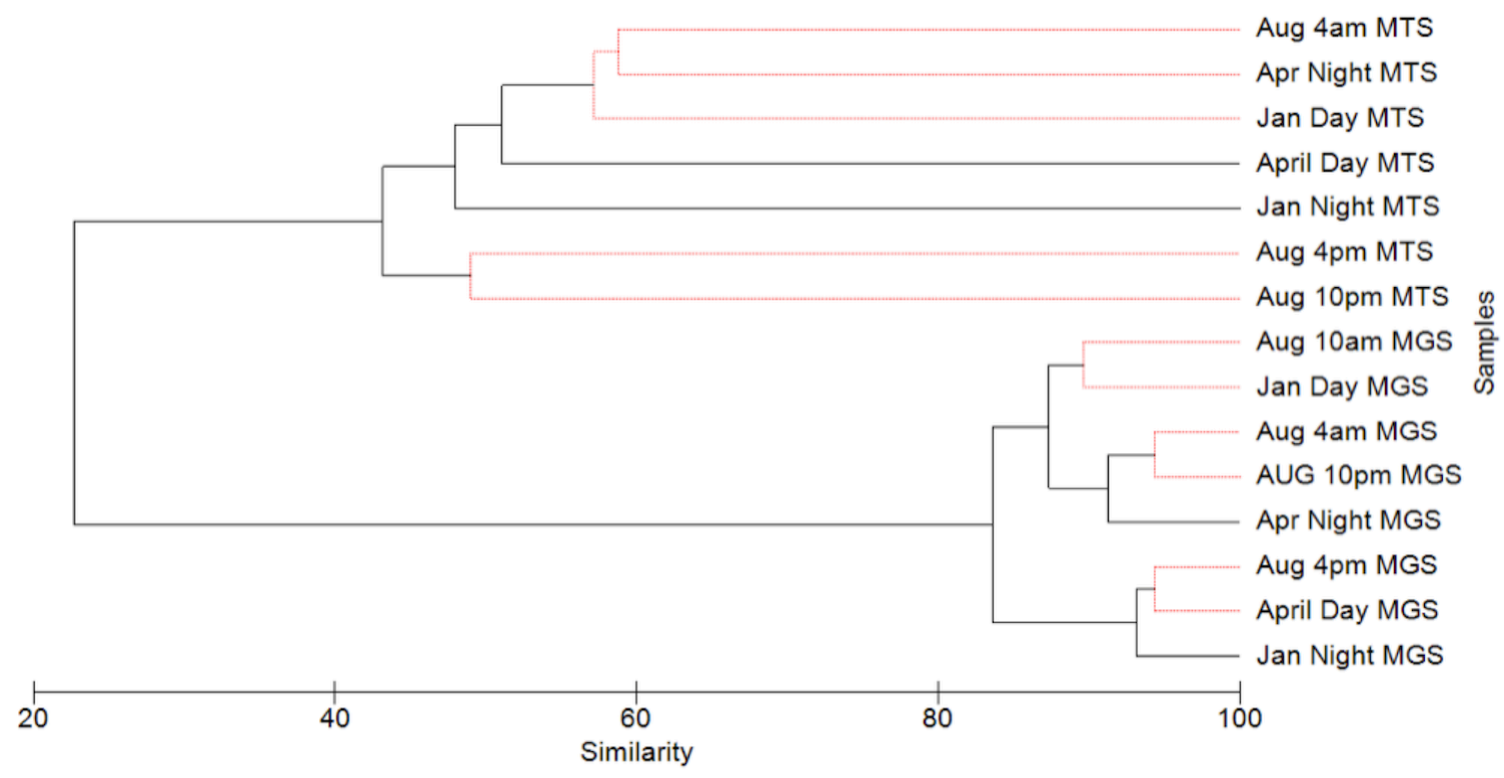

Figure 1. Group-average dendrogram showing relationship between all metagenomes and metatranscriptomes, based on comparison of annotated protein fragments via BLASTx using the SEED database in MGRAST for each dataset. MTS - metatranscriptome. MGS - metagenome. 
Table 1. Classification and general feature of 8 metagenome datasets according to the MIMS recommendations [5].

\begin{tabular}{llll}
\hline MIGS ID & Property & Term & Evidence code \\
\hline Current classification & $\begin{array}{l}\text { Metagenome ecological } \\
\text { metagenome marine } \\
\text { metagenome }\end{array}$ & TAS [6] \\
& &
\end{tabular}

Jan Day: 2008-01-28T15:30

Jan Night: 2008-01-28T19:00

Apr Day: 2008-04-22T16:00

5 Collection date

Apr Night: 2008

TAS [6]

Aug 4pm: 2008

Aug 10 pm: 2008

Aug 4 am: 2008

Aug 10 am: 2008

Jan Day: 50.2518:4.2089

Jan Night: 50.2611:4.2435

Apr Day: 50.2518:4.2089

6 Latitude Longitude

Apr Night: 50.2530:4.1875

NAS

Aug 4pm: 50.2518:4.2089

Aug 10 pm: 50.2545:4.1990

Aug 4 am: 50.2678:4.1990

Aug 10 am: 50.2665:4.1486

7

Depth

0

NAS

0

NAS

England

NAS

Coastal Marine

10 Environment

11a Environmental Package

See Table 2

29 Sample collection device or method

Large bore peristaltic filtration pump

Water filtered on to a $0.22 \mu \mathrm{m}$ Sterivex (Millipore) filter and then snap-frozen at $-80 \mathrm{C}$

$10 \mathrm{~L}$

Evidence codes - IDA: Inferred from Direct Assay (first time in publication); TAS: Traceable Author Statement (i.e., a direct report exists in the literature); NAS: Non-traceable Author Statement (i.e., not directly observed for the living, isolated sample, but based on a generally accepted property for the species, or anecdotal evidence). These evidence codes are from the Gene Ontology project [14]. If the evidence code is IDA, then the property was directly observed for a live isolate by one of the authors or an expert mentioned in the acknowledgements. 
Table 2. Environmental variables for each sampling occasion

\begin{tabular}{|c|c|c|c|c|c|c|c|c|c|}
\hline Property & & & & Measure & ement $^{a}$ & & & & \\
\hline Sample Collection date (MIGS-5) & 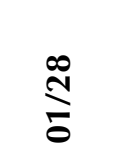 & 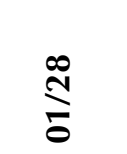 & 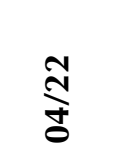 & 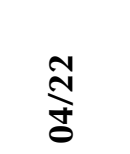 & 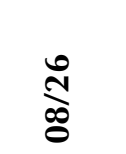 & 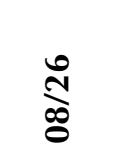 & 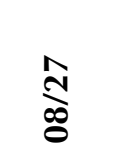 & 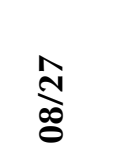 & 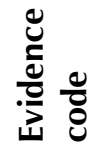 \\
\hline Sample collection time & $15: 38$ & $19: 30$ & $16: 00$ & $22: 00$ & $16: 00$ & 22.00 & 04:00 & 10:00 & \\
\hline Temperature $\left({ }^{\circ} \mathrm{C}\right)$ & 10.1 & 10.1 & 9.7 & 9.6 & 15.9 & 15.8 & 15.7 & 15.8 & IDA \\
\hline Density $\left(\mathrm{kg} \mathrm{m}^{-2}\right)$ & 1025.6 & 1026.3 & 1027.2 & 1027.1 & 1023.5 & 1024.3 & 1024.5 & 1024.4 & \\
\hline Salinity (PSU) & 33.3 & 34.2 & 35.1 & 35.0 & 32.1 & 33.0 & 33.3 & 33.2 & \\
\hline Chlorophyll a ( $\mu \mathrm{g} / \mathrm{L})$ & 0.8 & 0.9 & 2.2 & 1.3 & 9.2 & 8.2 & 9.8 & 11.9 & IDA \\
\hline Total Organic Nitrogen $(\mu \mathrm{mol} L-1)$ & 1.3 & 3.5 & 2.9 & 2.8 & 2.8 & 2.3 & 3.0 & 4.1 & IDA \\
\hline Total Organic Carbon $(\mu \mathrm{mol} L-1)$ & 33.2 & 38.2 & 27.2 & 19.4 & 26.8 & 26.5 & 22.0 & 23.7 & IDA \\
\hline $\mathrm{NO} 2+\mathrm{NO} 3(\mu \mathrm{mol} \mathrm{L}-1)$ & 10.9 & 10.0 & 4.0 & 3.8 & 0.1 & 0.1 & 0.9 & 0.1 & \\
\hline Ammonia ( $\mu \mathrm{mol} \mathrm{L}-1)$ & 0.0 & 0.0 & 0.5 & 0.3 & 0.1 & 0.1 & 0.1 & 0.1 & IDA \\
\hline $\operatorname{SRP}(\mu \mathrm{mol} L-1)$ & 0.5 & 0.5 & 0.4 & 0.3 & 0.0 & 0.1 & 0.0 & 0.1 & \\
\hline Silicate $(\mu \mathrm{mol} L-1)$ & 6.0 & 5.8 & 2.6 & 2.7 & 0.1 & 0.2 & 0.3 & 0.2 & \\
\hline
\end{tabular}

aSamples collected January - August, 2008. Evidence codes: MIGS-5: TAS [5].

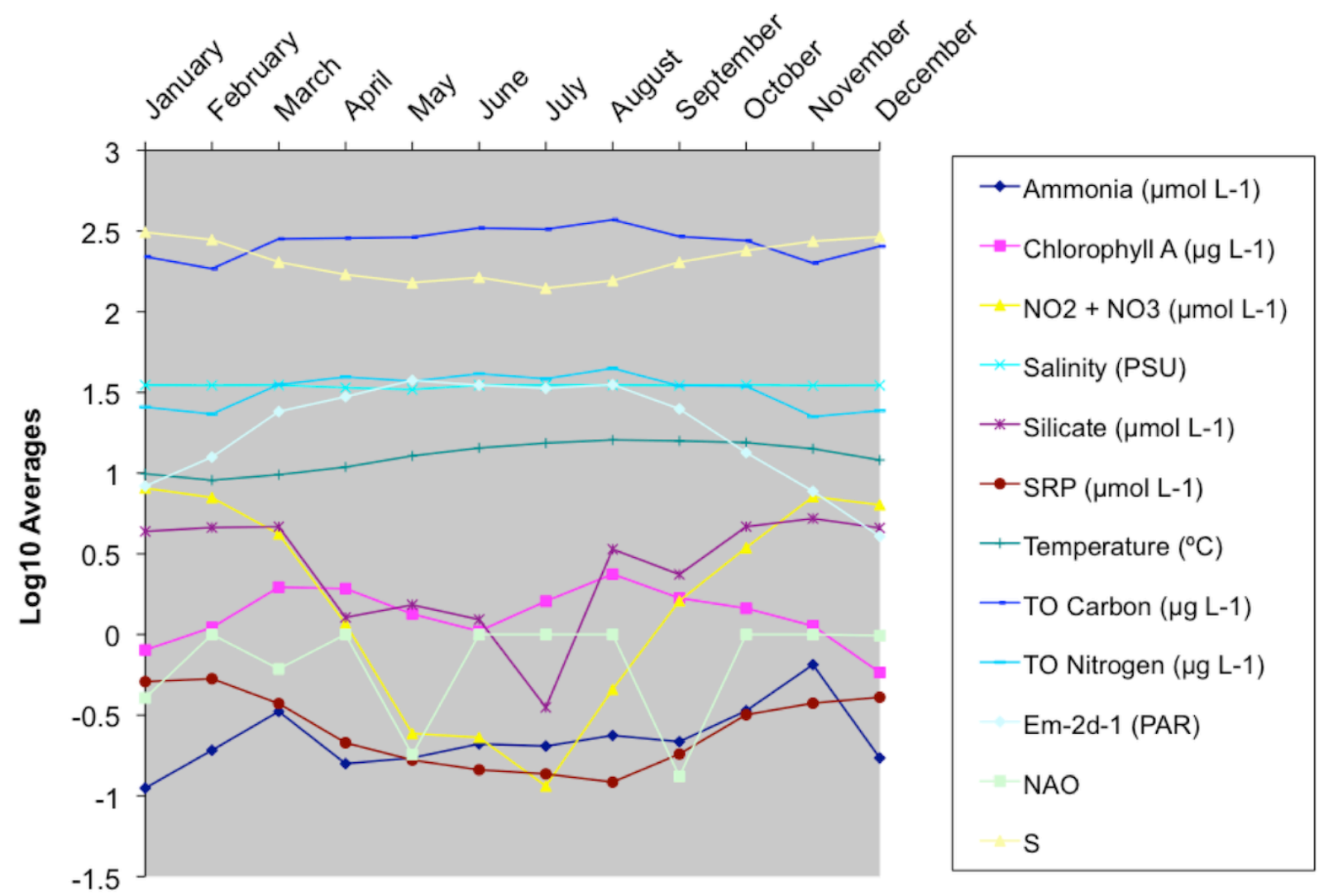

Figure 2. Monthly annual averages for all environmental parameters and species richness (S). TO - total organic; SRP - Soluble Reactive Phosphorous; PAR - Photosynthetically Active Radiation; NAO - North Atlantic Oscillation. Data taken from Gilbert et al., 2010. 


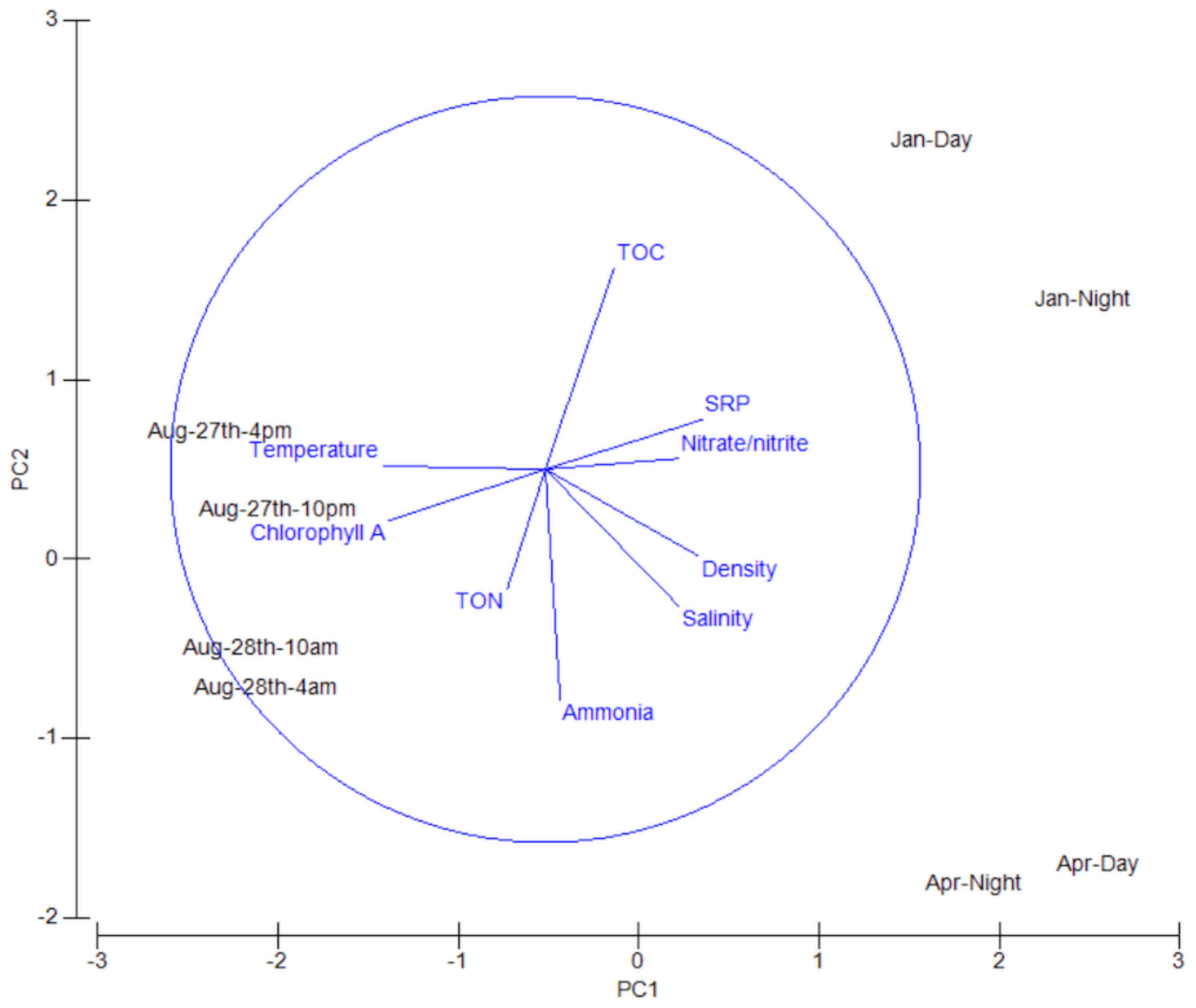

Figure 3. Principal component analysis of environmental variables showing the seasonal differences in variables outlined in Table 2. Classification and general features of the 15 datasets in accordance with the MIMS recommendations [5]

Table 3. Metagenome sequencing project information (MIMS compliance)

\begin{tabular}{|c|c|c|c|c|c|c|c|c|c|}
\hline MIGS ID & Property & 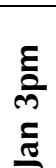 & 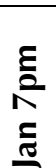 & $\begin{array}{l}\xi \\
\frac{\partial}{+} \\
\frac{a}{\alpha}\end{array}$ & $\begin{array}{l}\frac{\Sigma}{2} \\
\frac{2}{2} \\
\frac{2}{2}\end{array}$ & $\begin{array}{l}\frac{E}{2} \\
\frac{0}{+} \\
\frac{00}{2}\end{array}$ & 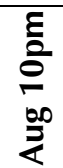 & 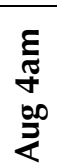 & 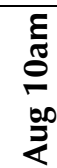 \\
\hline
\end{tabular}

$35 \quad$ library reads sequenced

32 nucleic acid extraction

43 sequencing method

46 Assembly

INSDC ID

GenBank Date of Release

GOLD ID
616,793 784,823 637,801 493,003 620,759 524,953 500,117 326,475

Gilbert et al. 2008

454 Titanium pyrosequencing (GS flx)

none

SRA009436

01-12-2009

GM00104 
Table 4. Metatranscriptome sequencing project information (MIMS compliance)

\begin{tabular}{|c|c|c|c|c|c|c|c|c|}
\hline MIGS ID & Property & $\begin{array}{l}\varepsilon \\
\text { के } \\
\stackrel{\equiv}{\Xi}\end{array}$ & $\begin{array}{l}\Sigma \\
\frac{1}{2} \\
\frac{\Xi}{\Xi}\end{array}$ & $\begin{array}{l}\varepsilon \\
\frac{\varepsilon}{+} \\
\frac{\bar{\alpha}}{<}\end{array}$ & $\begin{array}{l}\frac{\varepsilon}{2} \\
\frac{2}{2} \\
\frac{\grave{\alpha}}{k}\end{array}$ & $\begin{array}{l}\xi \\
\frac{\varepsilon}{+} \\
\frac{0}{20} \\
\frac{0}{2}\end{array}$ & 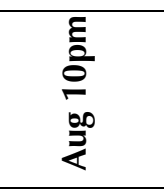 & 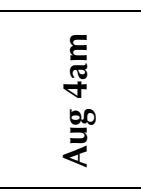 \\
\hline 35 & library reads sequenced & 139,880 & 130,826 & 124,925 & 147,492 & 139,375 & 193,254 & 154,865 \\
\hline 32 & nucleic acid extraction & \multicolumn{7}{|c|}{ Gilbert et al. 2008} \\
\hline 43 & sequencing method & \multicolumn{7}{|c|}{454 Titanium pyrosequencing (GS flx) } \\
\hline \multirow[t]{4}{*}{46} & Assembly & \multicolumn{7}{|c|}{ none } \\
\hline & INSDC ID & \multicolumn{7}{|c|}{ SRA009436 } \\
\hline & GenBank Date of Release & \multicolumn{7}{|c|}{ 01-12-2009 } \\
\hline & GOLD ID & \multicolumn{7}{|c|}{ GM00104 } \\
\hline
\end{tabular}

\section{Sampling and DNA isolation}

For the sampling, a minimal-impact surface buoy was deployed with a $7 \mathrm{~m}$ current drogue following a Lagrangian drift. Samples were taken at station L4 to represent three seasons and both day and night readings, as follows:

- Winter: January 28, at 3:00 pm and again at 7 pm (2 hours after sundown) at $50.2611 \mathrm{~N}: 4.2435 \mathrm{~W}$

- Spring: April 22, at $4 \mathrm{pm}$ and again at $10 \mathrm{pm}$ (one and a half hours after sundown) at $50.253 \mathrm{~N}: 4.1875 \mathrm{~W}$

- Summer: August 27, at 4 pm and again at $10 \mathrm{pm}$ (two hours after sundown) at $50.2545 \mathrm{~N}: 4.199 \mathrm{~W}$

- Summer: August 28, at 4 am (two hours before sunrise) at 50.2678N:4.1723W and at $10 \mathrm{am}$ at $50.2665 \mathrm{~N}: 4.1486 \mathrm{~W}$

The sampling technique involved the following steps: (1) collection of $20 \mathrm{~L}$ of seawater from the surface (0-2 m), (2) prefiltering through a $1.6 \mu \mathrm{m}$ GF/A filter (Whatmann), (3) passage of the filtrate through a $0.22 \mu \mathrm{m}$ Sterivex cartridge (Millipore) for a maximum of 30 minutes (approximately $10 \mathrm{~L}$ per Sterivex cartridge); (4) pump-drying and snap-freezing of the cartridges in liquid nitrogen, (5) barcoding [8] of the samples at the laboratory, and (6) storage at $-80^{\circ} \mathrm{C}$.
Both DNA and RNA then were isolated from each sample [2,9], barcoded, and stored at $-80^{\circ} \mathrm{C}$. DNA and mRNA-enriched cDNA were purified from the samples; for details, see [9].

\section{Metagenome sequencing and assembly}

The isolated DNA was used for metagenomic analysis, and the mRNA-enriched cDNA was used for metatranscriptomic pyrosequencing analysis. All DNA and cDNA were pyrosequenced on the GSFLX Titanium platform. No DNA assembly was carried out.

\section{Metagenome annotation}

The MG-RAST bioinformatics server [10] was used for annotating the metagenomic samples [113]. The data also were processed by using custom-written programming scripts on the BioLinux system [6] at the NERC Environmental Bioinformatics Centre unless otherwise indicated. In order to ensure high quality, the following sequences were removed from the pyrosequenced data: transcript fragments with $>10 \%$ nondetermined base pairs (Ns), fragments $<75 \mathrm{bp}$ in length, fragments with $>60 \%$ of any single base, and exact duplicates (resulting from aberrant dual reads during sequence analysis). So-called artificial duplicates in the metagenomic data (i.e., multiple reads that start at the same position; see, e.g., Gomez-Alvarez et al., 2009) were not removed, however, because of the possibility of their being natural; their removal would have precluded comparison with the metatranscriptomic data [12]. 
Table 5. Metagenome statistics

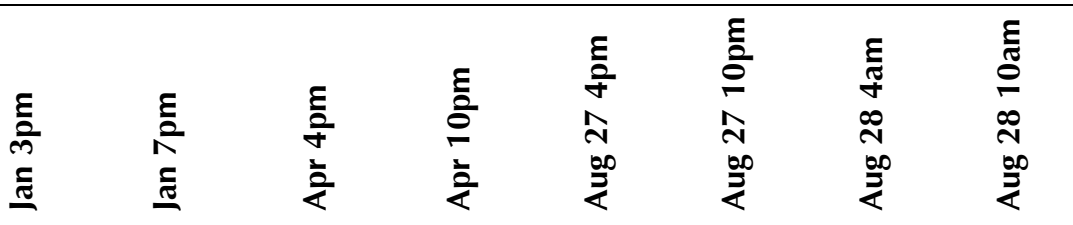

No. Original DNA Sequences

Predicted ORFs (>40aa pORFs)

No. of pORF clusters (95\%)

No. of pORF singletons (95\%)

No. of pORF 'families' (60\%)

No. of pORF singletons $(60 \%)$ $\begin{array}{llllllll}616,793 & 784,823 & 637,801 & 493,003 & 620,759 & 524,953 & 500,117 & 326,475\end{array}$

862,695 1,287,4121,003,799 745,305 986,269 846,209 779,951 491,330

$\begin{array}{llllllll}615,374 & 1,123,829 & 779,342 & 588,387 & 881,113 & 703,712 & 675,210 & 444,729\end{array}$

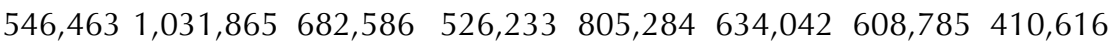

$\begin{array}{llllllll}423,674 & 1,031,904 & 678,547 & 528,213 & 801,760 & 637,542 & 620,403 & 419,461\end{array}$

$\begin{array}{llllllll}352,938 & 962,073 & 609,351 & 486,712 & 740,032 & 589,839 & 577,027 & 398,202\end{array}$

\begin{tabular}{|c|c|c|c|c|c|c|c|c|}
\hline \multicolumn{9}{|l|}{ Resampled pORFs (66529) } \\
\hline No. of pORF clusters (95\%) (66529) & 56337 & 64446 & 61187 & 59904 & 65601 & 63032 & 64729 & 65075 \\
\hline No. of pORF singletons (95\%) (66529) & 52891 & 63378 & 58691 & 57779 & 64818 & 61068 & 63359 & 63945 \\
\hline Good's Coverage (66529) & 20.50 & 4.74 & 11.78 & 13.15 & 2.57 & 8.21 & 4.76 & 3.88 \\
\hline $\begin{array}{l}\text { No. DNA seqs with } \\
\text { functional annotation }\end{array}$ & 122,936 & 291,953 & 258,658 & 164,249 & 283,761 & 196,369 & 196,972 & 126,392 \\
\hline $\begin{array}{l}\text { No. DNA seqs without } \\
\text { functional annotation (\%) }\end{array}$ & 493,857 & 492,870 & 379,143 & 328,754 & 336,998 & 328,584 & 303,145 & 200,083 \\
\hline $\begin{array}{l}\text { Percent DNA seqs without } \\
\text { functional annotation }\end{array}$ & $80 \%$ & $63 \%$ & $59 \%$ & $67 \%$ & $54 \%$ & $63 \%$ & $61 \%$ & $61 \%$ \\
\hline $\begin{array}{l}\text { No. DNA seqs with taxonomic } \\
\text { annotation }\end{array}$ & 190,326 & 417,920 & 349,888 & 241,541 & 379,911 & 288,356 & 304,003 & 186,421 \\
\hline \multicolumn{9}{|l|}{ Resampled sequencing effort $(\mathbf{1 8 6 , 4 2 1 )}$} \\
\hline $\begin{array}{l}\text { Number of archaeal sequences } \\
(186,421)\end{array}$ & 19,055 & 15,150 & 777 & 561 & 1,370 & 1,093 & 1,585 & 1,244 \\
\hline $\begin{array}{l}\text { Number of bacterial sequences } \\
(186,421)\end{array}$ & 161,899 & 146,911 & 182,850 & 180,674 & 182,717 & 176,825 & 180,725 & 182,332 \\
\hline
\end{tabular}

\section{Resampled pORFs (66529)}

No. of pORF singletons (95\%) (66529)

Good's Coverage (66529)

No. DNA seqs with

No. DNA seqs without

functional annotation (\%)

functional annotation

No. DNA seqs with taxonomic

Resampled sequencing effort $(\mathbf{1 8 6 , 4 2 1 )}$

Number of archaeal sequences

Number of bacterial sequences

The nucleic acid sequences were then compared with three major ribosomal RNA databases (SILVA, RDP II, and Greengenes - using the bacterial and archaeal 5S, 16S, and 23S and the eukaryotic $18 \mathrm{~S}$ and $25 \mathrm{~S}$ sequence annotator function of MG-RAST (e-value < 1 x 10-5; minimum length of alignment of $50 \mathrm{bp}$; minimum sequence nucleotide identity of $50 \%$ ). Reads annotated as rRNA were excluded. All subsequent reads were considered to be valid DNA or valid putative mRNA derived sequences and were annotated against the SEED database using MG-RAST (e-value $<1 \times 10-3$; minimum length of alignment of $50 \mathrm{bp}$; minimum sequence nucleotide identity of $50 \%$; Meyer et al., 2008). The result was an abundance matrix of functional genes and protein-derived predicted taxonomies across the DNA and mRNA samples.
The sequences also were translated using the techniques described by Gilbert et al. (2008) and Rusch et al. (2007) [9,13]. Predicted open reading frames (pORFs) having >40 amino acids were produced in all six reading frames. The CD-HIT program [15] was used to cluster the proteins from the datasets at 95\% amino acid identity over $80 \%$ of the length of the longest sequence in a cluster. The longest representative from each cluster then was clustered at $60 \%$ amino acid identity over $80 \%$ of the length of the longest sequence to group these sequences by protein families. Based on the relative abundance of each sample in a cluster, an abundance matrix was created using the output cluster files from CD-HIT that contained the original fasta sequences and headers for each sample (abundanceMatrix-twoStep.pl). 
Subsequently, protein clusters with $\leq 2$ representative pORFs were removed from the pORF matrix (MatrixParser.pv). In order to equalize the sequencing effort, all samples were randomly resampled (Daisychopper.pl) to the same number of pORFs or sequences across the clusters or functional/taxonomic SEED annotations.

\section{Metagenome properties}

Approximately 4.5 million combined microbial metagenomic reads were produced, comprising $\sim 1.9$ billion $\mathrm{bp}$, with an average read length of $\sim 350$ bp across the eight samples, ranging from
326,475 to 784,823 sequences [Table 5]. Seven metatranscriptomic datasets were also produced (the sample taken on August 28 at 10 am was lost in transit) totaling $\sim 1$ million sequences. After cleanup, 392,632 putative mRNA-derived sequences remained, totaling 159 million bp, with an average of $272 \mathrm{bp}$ per sequence. The effort per sample varied from 33,149 to 96,026 sequences [Table 6]. SEED annotations produced via MGRAST (Table 7 and Table 8 ranged from 20\% to $46 \%$ of each metagenomic dataset and from to $11 \%$ to $35 \%$ of the metatranscriptomic datasets.

Table 6. Metatranscriptome statistics

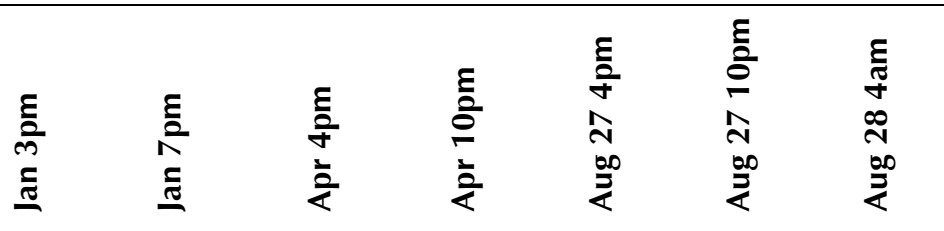

No. Original cDNA Sequences

No. of sequences following filtering***

No. mRNA following removal of rRNA

Predicted ORFs (>40aa pORFs)

No. of pORF clusters (95\%)

No. of pORF singletons (95\%)

No. of pORF 'families' (60\%)

No. of pORF singletons $(60 \%)$

\section{Resampled pORFs (66529)}

No. of pORF clusters (95\%) (66529)

No. of pORF singletons (95\%) (66529)

Good's Coverage (66529)

No. mRNA seqs with

functional annotation

No. mRNA seqs

without functional annotation

Percent DNA seqs

without functional annotation

No. mRNA seqs with

taxonomic annotation

\section{Resampled sequencing effort $(\mathbf{1 5 , 4 5 6 )}$}

Number of archaeal sequences $(15,456)$

Number of bacterial sequences $(15,456)$

\begin{tabular}{|c|c|c|c|c|c|c|}
\hline 139,880 & 130,826 & 124,925 & 147,492 & 139,375 & 193,254 & 15 \\
\hline 94,024 & 106,864 & 84,916 & 109,577 & 87,799 & 118,360 & 111,568 \\
\hline 61,831 & 96,026 & 41,378 & 53,413 & 33,149 & 51,829 & 55,006 \\
\hline 143,169 & 211,374 & 81,642 & 107,699 & 77,985 & 66,529 & 159,909 \\
\hline 98,871 & 78,278 & 35,648 & 51,088 & 28,167 & 24,136 & 68,080 \\
\hline 82,464 & 54,870 & 25,925 & 38,960 & 19,600 & 17,177 & 50,246 \\
\hline 84,598 & 45,049 & 19,131 & 37,628 & 15,146 & 12,735 & 41,480 \\
\hline 76,655 & 30,720 & 13,869 & 30,919 & 9,857 & 9,134 & 32,662 \\
\hline 31026 & 50354 & 30334 & 34217 & 24848 & 24136 & 33191 \\
\hline 23038 & 43687 & 22394 & 26840 & 17373 & 17177 & 25636 \\
\hline 65.37 & 34.33 & 66.34 & 59.66 & 73.89 & 74.18 & 61.47 \\
\hline 11,513 & 31,990 & 8,845 & 16,315 & 11,720 & 5,907 & 15,384 \\
\hline 50,318 & 64,036 & 32,533 & 37,098 & 21,429 & 45,922 & 39,622 \\
\hline $81 \%$ & $67 \%$ & $79 \%$ & $69 \%$ & $65 \%$ & $89 \%$ & $72 \%$ \\
\hline 29,521 & 30,778 & 20,899 & 26,398 & 15,456 & 29,605 & 38,304 \\
\hline 625 & 49 & 1 & 16 & 4 & 4 & 11 \\
\hline 13,633 & 11,926 & 13,702 & 8,449 & 14,469 & 15,071 & 14,803 \\
\hline
\end{tabular}


Table 7. Number of genes associated with the general SEED functional categories

\begin{tabular}{|c|c|c|c|c|c|c|c|c|}
\hline Subsystem Hierarchy 1 & $\begin{array}{c}\text { ह } \\
\text { ले } \\
\text { స్ }\end{array}$ & $\begin{array}{c}\frac{\Sigma}{2} \\
\tilde{N} \\
\tilde{\Xi}\end{array}$ & $\frac{\frac{\varepsilon}{\partial}}{\frac{\partial}{2}}$ & $\frac{\underline{z}}{\frac{z}{0}}$ & 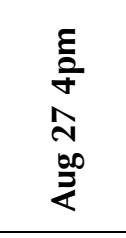 & 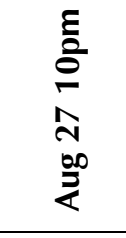 & 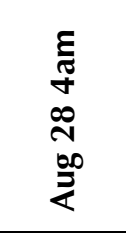 & 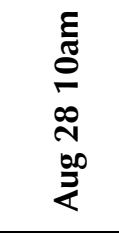 \\
\hline Amino Acids and Derivatives & 13,515 & 12,346 & 13,913 & 12,089 & 13,279 & 12,517 & 11,966 & 12,074 \\
\hline Carbohydrates & 14,181 & 13,087 & 14,884 & 13,829 & 14,801 & 13,929 & 13,258 & 13,780 \\
\hline Cell Division and Cell Cycle & 2,136 & 2,026 & 2,286 & 2,243 & 2,243 & 2,231 & 2,175 & 2,234 \\
\hline Cell Wall and Capsule & 5,632 & 5,363 & 5,336 & 6,051 & 5,553 & 5,674 & 6,079 & 6,347 \\
\hline Clustering-based subsystems & 18,051 & 17,585 & 19,425 & 19,647 & 19,055 & 19,441 & 20,434 & 19,860 \\
\hline $\begin{array}{l}\text { Cofactors, Vitamins, Prosthetic } \\
\text { Groups, Pigments }\end{array}$ & 8,497 & 7,675 & 8,188 & 8,606 & 8,142 & 8,227 & 8,582 & 8,001 \\
\hline DNA Metabolism & 5,461 & 5,331 & 5,191 & 5,559 & 5,321 & 5,717 & 5,824 & 5,855 \\
\hline Fatty Acids and Lipids & 2,165 & 1,919 & 1,883 & 1,891 & 1,955 & 2,025 & 1,960 & 1,934 \\
\hline Macromolecular Synthesis & 148 & 147 & 287 & 163 & 213 & 151 & 136 & 109 \\
\hline Membrane Transport & 2,764 & 2,322 & 2,839 & 2,375 & 2,606 & 2,507 & 2,234 & 2,234 \\
\hline Metabolism of Aromatic Compounds & 1,817 & 1,357 & 1,473 & 1,527 & 1,632 & 1,409 & 1,629 & 1,489 \\
\hline Miscellaneous & 381 & 367 & 448 & 423 & 417 & 446 & 454 & 393 \\
\hline Motility and Chemotaxis & 1,034 & 994 & 879 & 1,227 & 977 & 1,203 & 1,311 & 1,348 \\
\hline Nitrogen Metabolism & 668 & 688 & 587 & 574 & 747 & 718 & 628 & 660 \\
\hline Nucleosides and Nucleotides & 5,152 & 4,820 & 4,701 & 4,578 & 4,836 & 4,752 & 4,639 & 4,706 \\
\hline Phosphorus Metabolism & 1,796 & 1,706 & 1,747 & 1,926 & 1,832 & 1,958 & 2,085 & 1,879 \\
\hline Photosynthesis & 212 & 4,373 & 160 & 1,489 & 127 & 197 & 270 & 203 \\
\hline Potassium metabolism & 648 & 591 & 586 & 631 & 620 & 755 & 838 & 817 \\
\hline Protein Metabolism & 11,912 & 11,717 & 11,254 & 11,534 & 11,473 & 11,597 & 11,210 & 11,715 \\
\hline RNA Metabolism & 5,133 & 4,889 & 4,660 & 4,813 & 4,811 & 4,744 & 5,068 & 4,981 \\
\hline Regulation and Cell signaling & 1,196 & 1,127 & 1,400 & 966 & 1,356 & 1,360 & 1,076 & 1,056 \\
\hline Respiration & 5,298 & 8,480 & 5,455 & 5,570 & 5,432 & 5,579 & 4,926 & 4,994 \\
\hline Secondary Metabolism & 116 & 124 & 63 & 87 & 93 & 83 & 86 & 83 \\
\hline Stress Response & 2,497 & 2,133 & 2,338 & 2,419 & 2,306 & 2,524 & 2,508 & 2,605 \\
\hline Sulfur Metabolism & 1,604 & 1,354 & 1,673 & 1,430 & 1,446 & 1,240 & 1,320 & 1,317 \\
\hline Unclassified & 6,235 & 5,677 & 6,567 & 5,763 & 6,672 & 6,019 & 5,555 & 5,794 \\
\hline Virulence & 4,686 & 4,733 & 4,711 & 5,521 & 4,989 & 5,929 & 6,684 & 6,467 \\
\hline
\end{tabular}

\section{Highlights from the metagenome sequences}

In general, in the samples, metagenomes were more similar than metatranscriptomes. Photosynthesis genes showed both seasonal and diel changes: specifically, 10 times greater photosynthetic potential in winter than in summer and greater abundance at night in January and April. Gene fragments annotated to proteorhodopsin showed virtually no seasonal or diel fluctuations, however: only approximately $0.07 \%$ of the annotated functional profile from each sample. Other seasonal differences in metagenomic profiles included a considerably higher winter abundance (compared to spring or summer) of archaeal genes associated with lipid synthesis, thermosome chaperonins, RNA polymerase, small subunit ribosomal proteins, DNA replication, and rRNA modification. Diel differences were apparent among genes involved in respiratory metabolism, which were more abundant at night. 
Table 8. Number of transcripts associated with the general SEED functional categories

\begin{tabular}{|c|c|c|c|c|c|c|c|}
\hline Subsystem Hierarchy 1 & $\begin{array}{l}\varepsilon \\
\tilde{0} \\
\stackrel{0}{0} \\
\ddot{n} \\
\tilde{\Xi}\end{array}$ & $\begin{array}{l}\underline{2} \\
\hat{2} \\
\underline{\Xi}\end{array}$ & $\frac{\mathfrak{z}}{\frac{\partial}{\frac{\Sigma}{2}}}$ & $\frac{\varepsilon}{\frac{\varepsilon}{0}}$ & 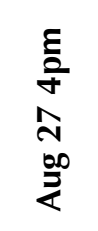 & 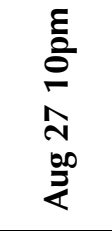 & 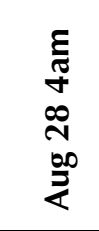 \\
\hline Amino Acids and Derivatives & 261 & 536 & 204 & 198 & 21 & 144 & 443 \\
\hline Carbohydrates & 886 & 1767 & 546 & 1302 & 530 & 1381 & 1256 \\
\hline Cell Division and Cell Cycle & 83 & 191 & 52 & 63 & 96 & 56 & 80 \\
\hline Cell Wall and Capsule & 154 & 353 & 317 & 297 & 153 & 113 & 221 \\
\hline Clustering-based subsystems & 641 & 657 & 294 & 451 & 111 & 157 & 427 \\
\hline $\begin{array}{l}\text { Cofactors, Vitamins, Prosthetic } \\
\text { Groups, Pigments }\end{array}$ & 215 & 457 & 130 & 248 & 24 & 13 & 469 \\
\hline DNA Metabolism & 102 & 108 & 83 & 122 & 24 & 26 & 85 \\
\hline Fatty Acids and Lipids & 84 & 28 & 17 & 27 & 0 & 28 & 10 \\
\hline Macromolecular Synthesis & 0 & 0 & 5 & 2 & 2 & 0 & 0 \\
\hline Membrane Transport & 44 & 19 & 237 & 83 & 2673 & 13 & 440 \\
\hline Metabolism of Aromatic Compounds & 47 & 6 & 16 & 4 & 0 & 24 & 14 \\
\hline Miscellaneous & 53 & 80 & 54 & 55 & 672 & 43 & 75 \\
\hline Motility and Chemotaxis & 40 & 10 & 438 & 58 & 3 & 8 & 180 \\
\hline Nitrogen Metabolism & 11 & 0 & 0 & 2 & 9 & 8 & 3 \\
\hline Nucleosides and Nucleotides & 144 & 87 & 42 & 48 & 4 & 13 & 56 \\
\hline Phosphorus Metabolism & 79 & 83 & 64 & 94 & 25 & 18 & 31 \\
\hline Photosynthesis & 67 & 0 & 17 & 2 & 0 & 1 & 0 \\
\hline Potassium metabolism & 29 & 13 & 3 & 13 & 4 & 2 & 7 \\
\hline Protein Metabolism & 439 & 95 & 129 & 625 & 81 & 112 & 172 \\
\hline RNA Metabolism & 1631 & 160 & 1813 & 702 & 907 & 2883 & 874 \\
\hline Regulation and Cell signaling & 65 & 136 & 16 & 354 & 30 & 18 & 41 \\
\hline Respiration & 174 & 20 & 26 & 97 & 125 & 31 & 109 \\
\hline Secondary Metabolism & 18 & 3 & 1 & 0 & 0 & 0 & 1 \\
\hline Stress Response & 100 & 175 & 42 & 229 & 5 & 43 & 56 \\
\hline Sulfur Metabolism & 42 & 18 & 19 & 14 & 13 & 11 & 40 \\
\hline Unclassified & 346 & 58 & 957 & 101 & 10 & 110 & 271 \\
\hline Virulence & 152 & 847 & 385 & 716 & 385 & 651 & 546 \\
\hline
\end{tabular}

The metatranscriptomic photosynthetic profiles were similar to those of the metagenomes in that photosynthesis genes were most abundant in January and virtually absent in August. Photosynthetic transcripts also were most abundant during the winter. On the other hand, unlike metagenomes, they were most abundant in the daytime in all months. Other seasonal differences in metatranscriptomic seasonal profiles included a greater abundance of transcripts related to membrane transport, especially amino acid transport, in summer when nutrients and dissolved organic material (DOM) are least abundant. The diel metatranscriptional profiles for January showed considerable difference in functions (in addition to photosynthesis); for example, transcripts relating to nitrogen cycling were most abundant during the day and were associated mainly with ammonification. Cell wall and capsule and cell division and cycle were upregulated at night, suggesting a nocturnal increase in cell division, potentially associated with the Cyanobacteria. Similarly, April samples showed a considerable up-regulation in RNA metabolism during the day, resulting primarily from an increase in group I intron and RNA polymerase transcripts. In August, transcripts with homology to membrane transport were upregulated during the day, while transcripts associated with motility and chemotaxis and with the synthesis of cofactors, vitamins, prosthetic groups, and pigments were considerably upregulated at night, suggesting that nocturnal motility and cellular activity (nucleotide and amino acid synthesis) were also upregulated. 


\section{Acknowledgments}

This work was funded by a grant from the Natural Environmental Research Council (NERC - NE/F00138X/1). The authors thank Neil Hall from the NERC / University of Liverpool Advanced Genomics Facility. This work was supported in part by the Office of Advanced Scientific Computing Research, Office of Science, U.S. Department of Energy, under Contract DE-AC02-06CH11357. The submitted manuscript has been created by UChicago Argonne, LLC, Operator of Argonne National Laboratory

\section{References}

1. Southward AJ, Langmead O, Hardman-Mountford NJ, Aiken J, Boalch GT, Dando PR, Genner MJ, Joint I, Kendall MA, Halliday NC, et al. Longterm oceanographic and ecological research in the Western English Channel. Adv Mar Biol 2004; 47:1-105. doi:10.1016/S0065-2881(04)47001-1

2. Gilbert JA, Field D, Swift P, Newbold L, Oliver A, Smyth T, Somerfield P, Huse S, Joint I. Seasonal succession of microbial communities in the Western English Channel using 16S rDNA-tag pyrosequencing. Environ Microbiol 2009; 11:31323139. PubMed doi:10.1111/j.14622920.2009.02017.x

3. Gilbert JA, Swift P, Somerfield P, Temperton B, Huse S, Smyth T, Field D. Seasonal succession and impact of environmental change on bacterial populations in the Western English Channel: A sixyear study. ISME J 2010; (In Review).

4. Siddorn JR, Allen JI, Uncles RJ. Heat, alt and tracer transport in the Plymouth Sound coastal region: a 3-D modeling study. I Mar Biol Assoc U K 2003; 83:673-682. doi:10.1017/S002531540300763Xh

5. Field D, Garrity G, Gray T, Morrison N, Selengut J, Sterk P, Tatusova T, Thomson N, Allen MJ, Angiuoli SV, et al. The minimum information about a genome sequence (MIGS) specification. Nat Biotechnol 2008; 26:541-547. PubMed doi:10.1038/nbt1360

6. Field D, Tiwari B, Booth T, Houten S, Swan D, Bertrand N, Thurston M. Open software for biologists: from famine to feast. Nat Biotechnol 2006; 24:801-803. PubMed doi:10.1038/nbt0706-801

7. Harris R. The L4 time-series: the first 20 years. J Plankton Res 2010; 32:577-583. doi:10.1093/plankt/fbq021

8. Booth T, Gilbert JA, Neufeld JD, Ball J, Thurston M, Chipman K, Joint I, Field D. Handlebar: a flexible, web-based inventory manager for handling
("Argonne"). Argonne, a U.S. Department of Energy Office of Science laboratory, is operated under Contract No. DE-AC02-06CH11357. The U.S. Government retains for itself, and others acting on its behalf, a paid-up nonexclusive, irrevocable worldwide license in said article to reproduce, prepare derivative works, distribute copies to the public, and perform publicly and display publicly, by or on behalf of the Government.

barcoded samples. Biotechniques 2007; 42:300302. PubMed doi: $10.2144 / 000112385$

9. Gilbert JA, Field D, Huang Y, Edwards R, Li W, Gilna $P$, Joint I. Detection of large numbers of novel sequences in the metatranscriptomes of complex marine microbial communities. PLOS ONE 2008; 3:e3042. PubMed doi:10.1371/journal.pone.0003042

10. Meyer F, Paarmann D, D'Souza M, Olson R, Glass EM, Kubal M, Paczian T, Stevens R, Wilke A, Wilkening J, Edwards RA. The Metagenomics RAST Server - a public resource for the automatic phylogenetic and functional analysis of metagenomes. BMC Bioinformatics 2008; 9:386. PubMed doi:10.1186/1471-2105-9-386

11. Gomez-Alvarez V, Teal TK, Schmidt TM. Systematic artifacts in metagenomes from complex microbial communitiesMetagenomes artifact. ISME J 2009; 3:1314-1317. PubMed doi:10.1038/ismej.2009.72

12. Niu B, Fu L, Sun S, Li W. Artificial and natural duplicates in pyrosequencing reads of metagenomic data. BMC Bioinformatics 2010; 11:187. PubMed doi:10.1186/1471-2105-11-187

13. Rusch DB, Halpern AL, Sutton $G$, Heidelberg KB, Williamson S, Yooseph S, Wu D, Eisen JA, Hoffman JM, Remington K, et al. The Sorcerer II Global Ocean Sampling Expedition: Northwest Atlantic through Eastern Tropical Pacific. PLoS Biol 2007; 5:e77. PubMed doi:10.1371/journal.pbio.0050077

14. Ashburner M, Ball CA, Blake JA, Botstein D, Butler H, Cherry JM, Davis AP, Dolinski K, Dwight SS, Eppig JT, et al. Gene Ontology: tool for the unification of biology. Nat Genet 2000; 25:2529. PubMed doi: $10.1038 / 75556$

15. Li W, Godzik A. Cd-hit: a fast program for clustering and comparing large sets of protein or nucleotide sequences. Bioinformatics 2006; 22:16581659. PubMed doi:10.1093/bioinformatics/btl158 